\title{
Primary Hypercholesterolemia, Carotid Atherosclerosis and Insulin Resistance Among Chinese
}

\author{
Kuo-Liong Chien $\cdot$ Chiau-Suong Liau $\cdot$ Ming-Fong Chen • \\ Yuan-Teh Lee · Jiann-Shing Jeng · Bao-Show Hwang • \\ Ta-Chen Su
}

Received: 5 June 2007/ Accepted: 30 October 2007/Published online: 15 December 2007

(c) AOCS 2007

\begin{abstract}
We investigated the genetic contributions to carotid atherosclerosis and insulin resistance in Chinese patients with primary hypercholesterolemia. A family study of probands from the outpatient clinics in patients with high low-density-lipoprotein cholesterol levels was conducted. A total of 62 families (360 subjects) underwent carotid ultrasonography and insulin resistance measurement. The correlation coefficients of carotid intima-media thickness (IMT) were high among spouse, parent-offspring, and sibling pairs $(0.39,0.38$ and 0.35 , respectively). All insulin indices and IMT had significant estimates of heritability, of which fasting insulin had the highest heritability $(0.410 \pm 0.104, \quad P=0.0001)$, followed by homeostasis model assessment (HOMA) $(0.395 \pm 0.108$, $P=0.0001)$. The estimated heritability of IMT was
\end{abstract}

\section{K.-L. Chien}

Institute of Preventive Medicine, School of Public Health,

National Taiwan University, Taipei, Taiwan

e-mail: klchien@ntu.edu.tw

K.-L. Chien · C.-S. Liau · M.-F. Chen · Y.-T. Lee · T.-C. Su Division of Cardiology, Department of Internal Medicine,

National Taiwan University Hospital, National Taiwan

University College of Medicine, Taipei, Taiwan

C.-S. Liau

Cardiovascular Center, Tzu-Chi Hospital, Hsin-Dian, Taipei,

Taiwan

J.-S. Jeng · B.-S. Hwang

Department of Neurology, National Taiwan University Hospital,

National Taiwan University College of Medicine, Taipei,

Taiwan

T.-C. Su $(\bowtie)$

Department of Environmental and Occupational Medicine,

National Taiwan University Hospital, Taipei, Taiwan

e-mail: tachensu@ntu.edu.tw significant $(0.185 \pm 0.103, P=0.025)$ but not of plaque score. Bivariate genetic coefficient between IMT and HOMA was $0.569 \pm 0.292$, while the environmental coefficient was $0.028 \pm 0.103$. The study confirms a relationship between insulin resistance and atherosclerosis and, in particular, between insulin resistance and the thickening of the arterial wall. Moreover, it shows that genetics influence both insulin resistance and atherosclerosis, implying that the management of insulin resistance may benefit the prevention of atherosclerotic disease in familial hypercholesterolemia.

Keywords Hypercholesterolemia - Insulin resistance . Carotid atherosclerosis · Family study

$\begin{array}{ll}\text { Abbreviations } \\ \text { CA } & \begin{array}{l}\text { Carotid atherosclerosis } \\ \text { CCA }\end{array} \\ \text { Common carotid artery } \\ \text { ECCA } & \begin{array}{l}\text { Extracranial carotid artery } \\ \text { FRSPH }\end{array} \\ & \begin{array}{l}\text { Familial-related severe primary hyper- } \\ \text { cholesterolemia }\end{array} \\ \text { HDL-C } & \text { High-density lipoprotein density } \\ \text { HOMA } & \text { Homeostasis model assessment } \\ \text { hs-CRP } & \text { High-sensitivity C-reactive protein } \\ \text { IMT } & \text { Intima-media thickness } \\ \text { LDL-C } & \text { Low-density lipoprotein cholesterol }\end{array}$

Introduction

Carotid atherosclerosis (CA) is a subclinical disease associated with cardiovascular disease and risk factors among ethnic Chinese [1, 2]. Although hypertension and related 
atherosclerotic risk factors are associated with the progression of CA, genetic factors also play an important role [3-5]. Previous familial aggregation studies showed significant heritability on the carotid artery intima-medial thickness (IMT) and plaque scores [4, 6, 7]. Moreover, genome-wide linkage results showed several genomic loci for determining carotid IMT trait [7-10].

Although there is evidence of genetic components for CA traits, there are several issues yet to be answered. First, the inconsistent genomic loci from genome-wide linkage results have implied genetic heterogeneity over genetic components controlling trait. Second, geographic and racial/ethnic variations of $\mathrm{CA}$ has been reported [5-7, 1115], however, little information is available in ethnic Chinese. Moreover, previous studies have shown that age, gender, insulin resistance, diabetes, metabolic syndrome, and family history were related to CA [9, 16-22]; however, association of these factors with $\mathrm{CA}$ have not been simultaneously examined in a family-based genetic study. In addition, hereditary factors have not been studied in ethnic Chinese. We designed a family-based genetic study that focused on ethnic Chinese, where the probands were recruited from hyperlipidemic clinics, in order to explore the genetic correlation and heritability of various CA traits. We also collected information on confounding factors, especially on metabolic syndrome and insulin resistance, to investigate the association with CA.

\section{Materials and Methods}

\section{Study Subjects}

This family study was conducted in hyperlipidemic outpatient clinics after developing standard procedures for recruiting probands and their family members in the university hospital [23]. Familial hypercholesterolemia screening was conducted in 2002 at the lipid clinic of the National Taiwan University Hospital and consecutive patients were recruited as proband. These probands were diagnosed as having familial-related severe primary hypercholesterolemia (FRSPH) on the basis of having low density lipoprotein-cholesterol (LDL-C) levels in themselves and in two or more first-degree relatives that were above $190 \mathrm{mg} / \mathrm{dL}$ and/or the presence of tendinous xanthomas within the kindred [24]. The spouses and firstdegree relatives were invited to participate in the study.

Individuals with some types of secondary hyperlipidemia, including nephrotic syndrome, obstructive liver disease, or hypothyroidism, and those who refused to provide informed consents, were excluded. The response rate was $90 \%$. The genetic basis of FRSPH is heterogeneous. The most common autosomal dominant form of $\mathrm{FH}$ is caused by mutation in the LDL receptor gene (LDLR) on chromosome 19 in band 19p13.2. There are a number of other less frequent forms of this disorder. They include type B hypercholesterolemia which is caused by liganddefective apolipoprotein B-100 (APOB), and autosomal dominant hypercholesterolemia 3 (HCHOLA3) which is caused by mutation in the PCSK9 gene. In addition, the differences in frequencies of specific mutations between populations should be considered. Instead of absence of genetic confirmation, we defined our study subjects as FRSPH [24]. All of the subjects received a detailed medical history review and physical examination.

The design was family study, based on the hypercholesterolemic patient as a proband and the first-degree relatives were recruited [25]. The study was approved by the Institutional Review Board of the National Taiwan University Hospital, and all of the subjects gave their informed consent. The procedure of collecting data was standardized, and was followed by the physicians and assistants in measuring the variables. We recruited a total of 360 members in the 62 families, which included three to six patients of hypercholesterolemia in each family, into our study. The subjects underwent examinations for anthropometric measurements, blood pressure, lipid profile, and carotid ultrasound measurements. Information on family history and medical diseases was obtained from each participant by trained assistants, using a structured questionnaire. Patient's socio-demographic characteristics, lifestyle, personal and family history of diseases, and history of hospitalization were also collected. Regular exercise habit was defined as the participants undertaking sports and leisure physical activity daily. Blood pressure was measured after resting for $10 \mathrm{~min}$, with the subjects in the sitting position, while body weight was measured using a calibrated balance. Body mass index was calculated as weight (kilogram) divided by height (meter) squared. The circumference of the smallest part of waist and the thickest part of the hip in the standing position were also measured.

\section{Blood Chemistry and Lipid Measurement}

Overnight fasting ( $>12 \mathrm{~h}$ ) blood samples were collected for measurements of glucose, total cholesterol, high-density lipoprotein cholesterol (HDL-C), and triglyceride by standard enzymatic methods with an automatic multi-channel chemical analyzer (Hitachi 7450, Hitachi Corp., Tokyo, Japan) in the central laboratory of the National Taiwan University Hospital. LDL-C value was calculated by the Friedewald's formula [26]. Blood samples for glucose analysis were drawn into glass test tubes, each containing $80 \mathrm{~mol} / \mathrm{L}$ fluoride/oxalate reagents. After centrifugation at $4{ }^{\circ} \mathrm{C}, 1,500 \mathrm{~g}$ for $10 \mathrm{~min}$, glucose levels were measured on 
the supernatant by enzymatic assay (Merck 3389 commercial kit, Germany) in an Eppendorf 5060 autoanalyzer.

For the following assays, blood samples were first centrifuged at $3,000 \mathrm{rpm}$ for $15 \mathrm{~min}$ within $30 \mathrm{~min}$ of collection, and then stored at $-70{ }^{\circ} \mathrm{C}$ until assayed. Serum insulin level was measured using a Microparticle Enzyme immunoassay (AxSYM Insulin, Abbott Diagnostic, Tokyo, Japan). Serum high-sensitivity C-reactive protein (hs-CRP) was measured using a chemi-luminescent enzyme-labeled immunometric assay (Immulite C-Reactive Protein, Diagnostic Products Co., Los Angeles).

\section{Insulin Resistance Indices}

Several measurements and parameters were used for insulin resistance indices. First, fasting insulin and glucose levels were used because fasting hyperinsulinemia was considered an important indicator of insulin resistance [27]. Second, homeostasis model assessment (HOMA) using the formula: [fasting insulin $(\mathrm{mU} / \mathrm{L}) \times$ fasting glucose $(\mathrm{mg} /$ $\mathrm{dL}) \times 0.05551] / 22.5$ was also used [28]. The numbers of metabolic syndrome components, defined by high blood pressure, high waist circumference, high fasting glucose, high triglyceride and low HDL-C status, were also utilized. Finally, we defined the metabolic syndrome status according to the criteria of the number of components more than or equal to three [29].

\section{Extracranial Carotid Artery Ultrasound Measurements}

The protocol and methods of CA measurements have been reported previously $[1,2,30]$. A Hewlett-Packard SONO 4500 ultrasound system (Andover, MA, USA) with a 3-11 $\mathrm{MHz}$ real-time B-mode scanner was used for evaluation, which included observation of the longitudinal and transverse views of the extracranial carotid artery (ECCA) bilaterally. An experienced ultrasonographer performed carotid ultrasonography while the patient was supine with the neck extended in a mild lateral rotation. Carotid endorgan disease was assessed by maximal IMT at the common carotid artery (CCA) and by ECCA plaque score. Two measurements of maximal IMT at CCA $0-20 \mathrm{~mm}$ proximal to the carotid bifurcation were obtained bilaterally. For future and subsequent off-line analysis, all scans were recorded on super-VHS videotape. Observers were blinded to the subjects' health status and risk factors. Intra-class correlation coefficients of intra-observer were about 0.70 0.87 for both sides of CCA IMT measurements, as reported previously [31]

Method for quantifying plaque score has been described previously $[1,31]$. Briefly, a focal thickening of IMT with
$>50 \%$ of thickness than the adjacent IMT was considered as an atherosclerotic plaque. A grade was assigned for each chosen segment: grade 0 for normal or no observable plaque; grade 1 for a small plaque with diameter stenosis $<30 \%$; grade 2 for a medium plaque with $30-49 \%$ diameter stenosis or multiple small plaques; grade 3 for one large plaque with $50-99 \%$ diameter stenosis or multiple plaques with at least one medium plaque; and grade 4 for $100 \%$ occlusion.

Extracranial carotid artery segments, including the proximal and distal CCA ( $>20$ and $0-20 \mathrm{~mm}$ distal to the bulb bifurcation, respectively), bulb, internal carotid artery, and external carotid artery, were examined bilaterally. The plaque score was calculated by adding up the plaque grades of ten segments of the ECCA. Reproducibility of the plaque grade scoring expressed good agreement with a kappa value of $0.70[1,31]$.

\section{Statistical Analysis}

Carotid atherosclerosis and insulin resistance indices, including fasting insulin, glucose and HOMA values, were presented in mean and standard deviation. We specified the above values by gender. Skewness and kurtosis of triglyceride, fasting glucose, insulin, and HOMA values were large due to a non-normal distribution. Logarithm-transformed variables were used for further genetic analysis but due to familial correlation, the statistical difference between genders was not tested. We have four IMT measurements at CCA for each participant, so mixed effect models can be applied in the analysis to increase the detection power in limited study samples. The mixed models were used to test the significance of contributions of IR indices to IMT of CCA and plaque score values in all family members [32].

The intra-familial correlation coefficients of carotid atherosclerosis traits were measured in different pairs, including spouse, parent-offspring, and siblings, by the FCOR program in SAGE [33]. Heritability estimates of carotid indices in the families were estimated by the variance component model, which was incorporated in the SOLAR software [34].

\section{Results}

Description of Study Participants

Compared with men, women tended to be older and to have higher total cholesterol, HDL, and LDL cholesterol levels, as well as lower waist circumference, uric acid, fasting insulin, HOMA values (Table 1). BMI and blood pressure 
Table 1 Demographic and insulin resistance index risk profiles in the family study (by gender)

\begin{tabular}{|c|c|c|c|c|c|}
\hline \multirow{2}{*}{\multicolumn{2}{|c|}{ Characteristics }} & \multicolumn{2}{|c|}{$\begin{array}{l}\text { Men } \\
(N=166)\end{array}$} & \multicolumn{2}{|c|}{$\begin{array}{l}\text { Women } \\
(N=194)\end{array}$} \\
\hline & & Mean & SD & Mean & SD \\
\hline \multicolumn{2}{|l|}{ Age (years) } & 39.2 & 17.5 & 45.5 & 17.1 \\
\hline \multicolumn{2}{|l|}{ Body mass index $\left(\mathrm{kg} / \mathrm{m}^{2}\right)$} & 23.8 & 4.1 & 22.7 & 3.7 \\
\hline \multicolumn{2}{|c|}{ Systolic blood pressure (mmHg) } & 113.4 & 14.9 & 114.4 & 18.8 \\
\hline \multicolumn{2}{|c|}{ Diastolic blood pressure $(\mathrm{mmHg})$} & 73.9 & 10.3 & 72.4 & 10.7 \\
\hline \multicolumn{2}{|l|}{ Waist circumference $(\mathrm{cm})$} & 83.3 & 11.8 & 77 & 10.3 \\
\hline \multicolumn{2}{|l|}{ Total cholesterol (mg/dL) } & 239.9 & 66.8 & 264 & 73.5 \\
\hline \multicolumn{2}{|l|}{ Triglyceride (mg/dL) } & 138 & 103.1 & 113.9 & 72.3 \\
\hline \multicolumn{2}{|l|}{ HDL cholesterol (mg/dL) } & 51.3 & 14.8 & 62.4 & 13.5 \\
\hline \multicolumn{2}{|l|}{ LDL cholesterol (mg/dL) } & 149.6 & 59.5 & 164.6 & 64.7 \\
\hline \multicolumn{2}{|l|}{ Serum creatinine $(\mathrm{mg} / \mathrm{dL})$} & 0.96 & 0.17 & 0.73 & 0.13 \\
\hline \multicolumn{2}{|l|}{ Uric acid (mg/dL) } & 7.58 & 1.67 & 5.83 & 1.63 \\
\hline \multicolumn{2}{|l|}{ Fasting glucose (mg/dL) } & 91.9 & 13 & 92.1 & 14.1 \\
\hline \multicolumn{2}{|l|}{ Fasting insulin (mU/L) } & 9.31 & 7.78 & 7.81 & 6.18 \\
\hline \multicolumn{2}{|l|}{ HOMA } & 2.17 & 1.88 & 1.83 & 1.54 \\
\hline \multicolumn{2}{|l|}{ Intima media thickness (mm) } & 0.06 & 0.01 & 0.06 & 0.02 \\
\hline \multicolumn{2}{|l|}{ Plaque score } & \multicolumn{2}{|l|}{0.12} & 0.14 & 0.27 \\
\hline Characteristics & $N$ & \multicolumn{2}{|c|}{$\%$} & $N$ & $\%$ \\
\hline Diabetes mellitus-yes & 2 & \multicolumn{2}{|r|}{1.2} & 5 & 2.6 \\
\hline Hyperlipidemia-yes & 88 & \multicolumn{2}{|c|}{53} & 117 & 60.3 \\
\hline Education group & & & & & \\
\hline$\leq 9$ years & 25 & & 15.3 & 43 & 23.2 \\
\hline$>9$ years & 138 & & 84.7 & 142 & 76.8 \\
\hline Job type & & & & & \\
\hline Labor work & 31 & & 18.9 & 45 & 23.4 \\
\hline House work & 1 & & 0.6 & 64 & 33.3 \\
\hline Nil & 25 & & 15.2 & 19 & 9.9 \\
\hline Business and office & 107 & & 65.2 & 64 & 33.3 \\
\hline Marital status & & & & & \\
\hline Couple & 93 & & 56.4 & 123 & 64.4 \\
\hline Single & 72 & & 43.6 & 68 & 35.6 \\
\hline Smoking & & & & & \\
\hline Current & 40 & & 24.1 & 9 & 4.7 \\
\hline Nil & 113 & & 68.1 & 183 & 94.8 \\
\hline Quit & 13 & & 7.8 & 1 & 0.5 \\
\hline Alcohol drinking & & & & & \\
\hline Current & 43 & & 27 & 4 & 2.1 \\
\hline Nil & 114 & & 71.7 & 181 & 95.8 \\
\hline Quit & 2 & & 1.3 & 4 & 2.1 \\
\hline Exercise & & & & & \\
\hline Non-regular & 63 & & 38.2 & 81 & 42.4 \\
\hline Regular & 102 & & 61.8 & 110 & 57.6 \\
\hline Metabolic syndrome & & & & & \\
\hline No & 127 & & 84.1 & 140 & 82.8 \\
\hline Yes & 24 & & 15.9 & 29 & 17.2 \\
\hline
\end{tabular}

in women were comparable with those in men. Carotid atherosclerosis indices, including IMT and plaque scores, were similar between genders. Most patients had hyperlipidemic status, educational level $\geq 9$ years, and regular exercise habits. Their jobs were business and official work. Men had higher proportions of smoking and alcohol drinking habits. The prevalence rates of metabolic syndrome, defined by AHA/NHLBI criteria, were $15.9 \%$ in men and $17.2 \%$ in women.

Predictor of Carotid Atherosclerosis by Insulin Resistance Indices

We estimated the effects of association between carotid atherosclerosis and various insulin resistance indices (Table 2) using the mixed models. In the univariate model, all insulin resistance indices were associated well with IMT. The magnitude of effects was reduced somewhat after age and gender adjustment. In the multivariate models that incorporated age, gender, obesity, married status, smoking, drinking, education and sport habits, the insulin resistance indices of fasting insulin, glucose, HOMA were still significant predictors for IMT values $(P=0.030$, 0.003 , and 0.019, respectively). Metabolic syndrome component numbers and status were non-significant predictors for IMT in multivariate models. For plaque scores, most of the estimated parameters were not statistically significant in the multivariate models, and only fasting glucose was a significant predictor for plaque score $(0.00325 \pm 0.00107, P=0.003)$. Insulin resistance indices were associated with IMT values, not with plaque scores.

\section{Familial Correlation and Heritability}

In all of the family members, there were 45 spouse pairs, 266 parent-offspring pairs, and 236 sibling pairs. Familial correlation coefficients and heritability estimates were shown in Table 3. The coefficients of parent-offspring and sibling pairs were higher than those of spouses in insulin resistance and carotid atherosclerosis indices. Among these, the IMT coefficients were higher among spouse, parent-offspring, and sibling pairs (0.389, 0.376, and 0.354 , respectively). Gender specific parent-offspring correlation coefficients varied much: mothers had higher coefficients than fathers in insulin resistance indices (0.426 in mother-daughter, 0.322 in mother-son, 0.043 in father-daughter, and -0.083 in father-son for HOMA, respectively).

For IMT values, the coefficients were equal in motherdaughter and father-son pairs (0.454 and 0.489, respectively). The coefficients of plaque scores varied much and 
Table 2 Estimated parameters and standard errors of various insulin resistance indices for the intima media thickness and plaque scores in the study population, after adjusted for familial correlation by mixed models

\begin{tabular}{|c|c|c|c|c|c|c|}
\hline & \multicolumn{3}{|c|}{ Intima media thickness, $\mathrm{mm}$} & \multicolumn{3}{|l|}{ Plaque score } \\
\hline & Estimated parameter & Standard error & $P$ value & Estimated parameter & Standard error & $P$ value \\
\hline \multicolumn{7}{|l|}{ 1. Univariate } \\
\hline Fasting insulin, mU/L & 0.00018 & 0.00013 & 0.157 & 0.00154 & 0.00228 & 0.499 \\
\hline Fasting glucose, mg/dL & 0.00033 & 0.00006 & $<0.0001$ & 0.00602 & 0.00108 & $<0.0001$ \\
\hline HOMA & 0.00113 & 0.00051 & 0.029 & 0.01366 & 0.00937 & 0.146 \\
\hline Metabolic syndrome & 0.00748 & 0.00105 & $<0.0001$ & 0.09434 & 0.02052 & $<0.0001$ \\
\hline \multicolumn{7}{|l|}{ 2. Age, sex adjusted } \\
\hline Fasting insulin, mU/L & 0.00018 & 0.00009 & 0.059 & 0.00175 & 0.00194 & 0.367 \\
\hline Fasting glucose, $\mathrm{mg} / \mathrm{dL}$ & 0.00011 & 0.00005 & 0.022 & 0.00285 & 0.00099 & 0.004 \\
\hline HOMA & 0.00081 & 0.00038 & 0.036 & 0.00988 & 0.00795 & 0.216 \\
\hline Metabolic syndrome & 0.00215 & 0.00089 & 0.016 & 0.01406 & 0.01982 & 0.479 \\
\hline \multicolumn{7}{|l|}{ 3. Multivariate adjusted ${ }^{\mathrm{a}}$} \\
\hline Fasting insulin, mU/L & 0.00021 & 0.00010 & 0.030 & 0.00240 & 0.00194 & 0.217 \\
\hline Fasting glucose, $\mathrm{mg} / \mathrm{dL}$ & 0.00016 & 0.00005 & 0.003 & 0.00325 & 0.00107 & 0.003 \\
\hline HOMA & 0.00092 & 0.00039 & 0.019 & 0.01203 & 0.00802 & 0.135 \\
\hline Metabolic syndrome & 0.00154 & 0.00091 & 0.094 & -0.00632 & 0.02037 & 0.757 \\
\hline
\end{tabular}

${ }^{a}$ Adjusted covariates in multivariate models included age, gender, obesity, married, smoking, drinking, education, and sport habits

high in sibling and spouse pairs. All insulin resistance indices and IMT had significant heritability estimates, and the highest heritability values were fasting insulin $(0.410 \pm 0.104, P=0.0001)$, then HOMA $(0.395 \pm 0.108$, $P=0.0001)$. The estimated heritability of IMT was significant $(0.185 \pm 0.103, P=0.025)$ but the plaque score heritability was not statistically different.

Genetic and environmental correlation between CA and insulin resistance in bivariate models showed that genetic correlation coefficients were higher (0.999 in HOMA vs. insulin and 1.00 in IMT vs. plaque score). Genetic coefficients between IMT and HOMA were $0.569 \pm 0.292$ and environmental coefficients were $0.028 \pm 0.103$.

\section{Discussion}

The study confirms a relationship between insulin resistance and atherosclerosis and, in particular, between insulin resistance and the thickening of the arterial wall. Moreover,

Table 3 Familial correlation coefficients, heritability and standard error estimates for insulin resistance indices and carotid atherosclerosis in the study population

\begin{tabular}{|c|c|c|c|c|c|c|}
\hline \multirow[t]{2}{*}{ Familial pair type } & \multirow[t]{2}{*}{ Number of pairs } & \multicolumn{5}{|c|}{ Correlation coefficients } \\
\hline & & Fasting insulin & Fasting glucose & HOMA & Intima media thickness & Plaque score \\
\hline Spouse & 45 & 0.140 & 0.013 & 0.155 & 0.389 & 0.451 \\
\hline Parent-offspring & 266 & 0.219 & 0.056 & 0.222 & 0.376 & 0.135 \\
\hline Sibling & 236 & 0.255 & 0.188 & 0.201 & 0.354 & 0.301 \\
\hline Mother-daughter & 84 & 0.410 & 0.015 & 0.426 & 0.454 & 0.016 \\
\hline Mother-son & 89 & 0.316 & 0.307 & 0.322 & 0.349 & 0.256 \\
\hline Father-daughter & 44 & 0.010 & 0.307 & 0.043 & 0.172 & 0.167 \\
\hline Father-son & 49 & -0.079 & -0.282 & -0.083 & 0.489 & 0.080 \\
\hline Sister-sister & 71 & -0.032 & 0.082 & -0.075 & 0.362 & 0.363 \\
\hline Sister-brother & 118 & 0.319 & 0.297 & 0.278 & 0.371 & 0.392 \\
\hline Brother-brother & 47 & 0.259 & 0.414 & 0.262 & 0.293 & -0.048 \\
\hline Heritability & & 0.410 & 0.370 & 0.395 & 0.185 & 0.062 \\
\hline Standard error & & 0.104 & 0.181 & 0.108 & 0.103 & 0.085 \\
\hline$P$ value & & 0.0001 & 0.018 & 0.0001 & 0.025 & 0.212 \\
\hline
\end{tabular}


it shows that genetics influence both insulin resistance and atherosclerosis. This family-based study on ethnic Chinese clearly demonstrated the association between insulin resistance indices and $\mathrm{CA}$, which showed significant heritability values among the study subjects. The more novel component of this study is the effort to consider joint genetic determinants of IMT and insulin resistance.

The relationship between insulin resistance indices and IMT had been previously demonstrated among young Italian women [16]. Although some studies did not show an association of insulin resistance and carotid IMT [35, 36], most studies have shown a positive relation between insulin resistance and carotid IMT [16, 37, 38]. The implications of the study involve several aspects. First, as ascertained from hyperlipidemic patients, family members showed significant familial aggregation of insulin resistance indices and CA. These findings also echoed results in other population-based studies [18, 22, 39, 40]. Our study differentiated two traits of CA, i.e., IMT and plaque score. IMT had higher heritability and familial correlation than plaque score in the study population, while advanced plaque score did not have significant heritability, which were different from those of Mexican American families [10]. Moreover, heritability for IMT was higher for the spouse than for the parent-offspring and sibling, which implied that common environmental factors were as important as genetic factors. Insulin resistance coefficients were ten times higher in the mother-daughter than in the fatherdaughter, indicating that maternal transmission of glucose metabolism and insulin resistance was much more important than paternal transmission. The hypothesis of excess maternal transmission in diabetes and related traits has been postulated [41]. The mechanisms of female predominance in terms of transmission were proposed as the unique maternal genetic and environmental effects [42], with gestational diabetes having a strong effect on offspring diabetic risk [43]. Our data showed a higher heritability from maternal lineage than paternal one.

We emphasized the relationship between insulin resistance indices and $\mathrm{CA}$, which were taken into consideration together. For predicting $\mathrm{CA}$, various insulin resistance indices, such as fasting insulin, glucose, HOMA, and metabolic syndrome components, had positive effects on IMT, but not plaque scores. High plaque scores were an indicator of more advanced $\mathrm{CA}$, which was evident in men over 40 years of age and in post-menopausal women. With regards to plaque scores, this study did not show a significant heritability between family members whom only IMT values were affected by markers of insulin resistance. The relative young age in these families, with a mean age of 39 and 45 years in men and women, respectively, might be the reason of lack of heritability. As age increases, the association of plaque severity with insulin resistance might be more apparent [10, 22, 39, 40, 44]. Our participants were in a higher education and official job and tended to maintain a healthy lifestyle, which may blunt the progress of carotid atherosclerosis. Social class disparities in atherosclerosis were observed among general population: adults in US with higher social class keeping a healthier lifestyle, such as exercise and smoking cessation [45].

The strength of our study includes the complete measurements of insulin resistance indices and $\mathrm{CA}$, and the focus on hypercholesterolemic proband populations. The study limitation is that we cannot separate the pure genetic factors from shared common household factors in the estimates of heritability. The high genetic correlation observed seems counterintuitive since this parameter requires that there is a significant heritability to both traits separately. Relatively small sample size in this study may limit the power, especially after being stratified by different relative pairs.

In conclusion, our finding confirms significant heritability for IMT and HOMA, in line with many previous studies, and demonstrates a moderately high genetic correlation between these two traits, implying that common genes contribute to variation in both traits. The association and heritability of insulin resistance and CA were evident among hypercholesterolemic proband families. The results imply that potential pleiotropy exists among these traits. As knowledge of clinical phenotypes and the technical improvement in post-human genome era, the genetic locus of complex traits, such as insulin resistance and CA will be revealed in the near future.

Acknowledgments This study was partially funded by NSC 942314-B-002-300 grant from the National Science Council, Taiwan, and the National Taiwan University Hospital.

\section{References}

1. Su TC, Jeng JS, Chien KL, Sung FC, Hsu HC, Lee YT (2001) Hypertension status is the major determinant of carotid atherosclerosis: a community-based study in Taiwan. Stroke 32:22652271

2. Su TC, Lee YT, Chou S, Hwang WT, Chen CF, Wang JD (2006) Twenty-four-hour ambulatory blood pressure and duration of hypertension as major determinants for intima-media thickness and atherosclerosis of carotid arteries. Atherosclerosis 184:151156

3. Lange LA, Bowden DW, Langefeld CD et al (2002) Heritability of carotid artery intima-medial thickness in type 2 diabetes. Stroke 33:1876-1881

4. Moskau S, Golla A, Grothe C, Boes M, Pohl C, Klockgether T (2005) Heritability of carotid artery atherosclerotic lesions: an ultrasound study in 154 families. Stroke 36:5-8

5. Xiang AH, Azen SP, Buchanan TA et al (2002) Heritability of subclinical atherosclerosis in Latino families ascertained through a hypertensive parent. Arterioscler Thromb Vasc Biol 22:843848 
6. Sayed-Tabatabaei FA, van Rijn MJ, Schut AF et al (2005) Heritability of the function and structure of the arterial wall: findings of the Erasmus Rucphen family (ERF) study. Stroke 36:23512356

7. Pankow JS, Heiss G, Evans GW et al (2004) Familial aggregation and genome-wide linkage analysis of carotid artery plaque: the NHLBI family heart study. Hum Hered 57:80-89

8. Fox CS, Cupples LA, Chazaro I et al (2004) Genome-wide linkage analysis for internal carotid artery intimal medial thickness: evidence for linkage to chromosome 12. Am J Hum Genet $74: 253-261$

9. Wang TJ, Nam BH, D'Agostino RB et al (2003) Carotid intimamedia thickness is associated with premature parental coronary heart disease: the Framingham heart study. Circulation 108:572576

10. Hunt KJ, Duggirala R, Goring HH et al (2002) Genetic basis of variation in carotid artery plaque in the San Antonio family heart study. Stroke 33:2775-2780

11. Juonala M, Viikari JS, Kahonen M et al (2005) Geographic origin as a determinant of carotid artery intima-media thickness and brachial artery flow-mediated dilation: the cardiovascular risk in Young Finns study. Arterioscler Thromb Vasc Biol 25:392-398

12. Tatsukawa M, Sawayama Y, Maeda N et al (2004) Carotid atherosclerosis and cardiovascular risk factors: a comparison of residents of a rural area of Okinawa with residents of a typical suburban area of Fukuoka, Japan. Atherosclerosis 172:337-343

13. Fox CS, Polak JF, Chazaro I et al (2003) Genetic and environmental contributions to atherosclerosis phenotypes in men and women: heritability of carotid intima-media thickness in the Framingham heart study. Stroke 34:397-401

14. Juo SH, Lin HF, Rundek T et al (2004) Genetic and environmental contributions to carotid intima-media thickness and obesity phenotypes in the Northern Manhattan family study. Stroke 35:2243-2247

15. North KE, MacCluer JW, Devereux RB et al (2002) Heritability of carotid artery structure and function: the Strong heart family study. Arterioscler Thromb Vasc Biol 22:1698-1703

16. De PG, Ciccone M, Pannacciulli N et al (2000) Lower insulin sensitivity as an independent risk factor for carotid wall thickening in normotensive, non-diabetic, non-smoking normal weight and obese premenopausal women. Int J Obes Relat Metab Disord 24:825-829

17. Hunt KJ, Williams K, Rivera D et al (2003) Elevated carotid artery intima-media thickness levels in individuals who subsequently develop type 2 diabetes. Arterioscler Thromb Vasc Biol 23:1845-1850

18. Kitamura A, Iso $\mathrm{H}$, Imano $\mathrm{H}$ et al (2004) Carotid intima-media thickness and plaque characteristics as a risk factor for stroke in Japanese elderly men. Stroke 35:2788-2794

19. Larsen JR, Brekke M, Bergengen L et al (2005) Mean HbA1c over 18 years predicts carotid intima media thickness in women with type 1 diabetes. Diabetologia 48:776-779

20. Pannacciulli N, De PG, Ciccone M, Rizzon P, Giorgino F, Giorgino R (2003) Effect of family history of type 2 diabetes on the intima-media thickness of the common carotid artery in normal-weight, overweight, and obese glucose-tolerant young adults. Diabetes Care 26:1230-1234

21. Sigurdardottir V, Fagerberg B, Hulthe J (2004) Preclinical atherosclerosis and inflammation in 61-year-old men with newly diagnosed diabetes and established diabetes. Diabetes Care 27:880-884

22. Tzou WS, Douglas PS, Srinivasan SR et al (2005) Increased subclinical atherosclerosis in young adults with metabolic syndrome: the Bogalusa heart study. J Am Coll Cardiol 46:457-463

23. Chien KL, Chen WJ, Hsu HC, Su TC, Chen MF, Lee YT (2006) Major gene effects on apolipoprotein B concentrations in families of adolescents-Results from a community-based study in Taiwan. Clin Chim Acta 365:194-199

24. Lin LY, Liau CS, Yang WS, Su TC (2005) Decreased serum adiponectin in adolescents and young adults with familial primary hypercholesterolemia. Lipids 40:163-167

25. Chien KL, Hsu HC, Su TC, Chen MF, Lee YT (2006) Heritability and major gene effects on left ventricular mass in the Chinese population: a family study. BMC Cardiovasc Disord 6:37

26. Friedewald WT, Levy RI, Fredrickson DS (1972) Estimation of the concentration of low-density lipoprotein cholesterol in plasma, without use of the preparative ultracentrifuge. Clin Chem 18:499-502

27. Chien KL, Lee YT, Sung FC, Hsu HC, Su TC, Lin RS (1999) Hyperinsulinemia and related atherosclerotic risk factors in the population at cardiovascular risk: a community-based study. Clin Chem 45:838-846

28. Matthews DR, Hosker JP, Rudenski AS, Naylor BA, Treacher DF, Turner RC (1985) Homeostasis model assessment: insulin resistance and beta-cell function from fasting plasma glucose and insulin concentrations in man. Diabetologia 28:412-419

29. NCEP (2002) Third Report of the National Cholesterol Education Program (NCEP) Expert Panel on Detection, Evaluation, and Treatment of High Blood Cholesterol in Adults (Adult Treatment Panel III) final report. Circulation 106:3143-3421

30. Su TC, Jeng JS, Wang JD et al (2006) Homocysteine, circulating vascular cell adhesion molecule and carotid atherosclerosis in postmenopausal vegetarian women and omnivores. Atherosclerosis 184:356-362

31. Su TC, Jeng JS, Chien KL, Torng PL, Sung FC, Lee YT (1999) Measurement reliability of common carotid artery intima-media thickness by ultrasonographic assessment. J Med Ultrasound 7:73-79

32. Liang KY, Zeger SL (1993) Regression analysis for correlated data. Annu Rev Public Health 14:43-68

33. SAGE (1997) Statistical analysis for genetic epidemiology, Release 3.1. Computer program package available from the Department of Epidemiology and Biostatistics, Rammelkamp Center for Education and Research, Metro Health Campus, Case Western Reserve University, Cleveland. In.

34. Almasy L, Blangero J (1998) Multipoint quantitative-trait linkage analysis in general pedigrees. Am J Hum Genet 62:1198-1211

35. Larsson H, Berglund G, Ahren B (2003) Insulin sensitivity, insulin secretion, and glucose tolerance versus intima-media thickness in nondiabetic postmenopausal women. J Clin Endocrinol Metab 88:4791-4797

36. Snehalatha C, Vijay V, Suresh MR et al (2001) Lack of association of insulin resistance and carotid intimal medial thickness in non-diabetic Asian Indian subjects. Diabetes Metab Res Rev $17: 444-447$

37. Golden SH, Folsom AR, Coresh J, Sharrett AR, Szklo M, Brancati F (2002) Risk factor groupings related to insulin resistance and their synergistic effects on subclinical atherosclerosis: the atherosclerosis risk in communities study. Diabetes 51:3069-3076

38. Yang B, Li TD, Wang JS, Zhi G, Jin WS, Xu Y (2005) Insulin resistance and carotid atherosclerosis in 221 patients with potential hyperglycemia. Chin Med Sci J 20:108-111

39. Joakimsen O, Bonaa KH, Stensland-Bugge E, Jacobsen BK (1999) Age and sex differences in the distribution and ultrasound morphology of carotid atherosclerosis: the Tromsö Study. Arterioscler Thromb Vasc Biol 19:3007-3013

40. Mathiesen EB, Joakimsen O, Bonaa KH (2001) Prevalence of and risk factors associated with carotid artery stenosis: the Tromsö study. Cerebrovasc Dis 12:44-51

41. Lin RS, Lee WC, Chou P, Fu CC (1994) Maternal role in type 2 diabetes mellitus: indirect evidence for a mitochondrial inheritance. Int J Epidemiol 23:886-890 
42. Mahtani MM, Widen E, Lehto M et al (1996) Mapping of a gene for type 2 diabetes associated with an insulin secretion defect by a genome scan in Finnish families. Nat Genet 14:90-94

43. McLean M, Chipps D, Cheung NW (2006) Mother to child transmission of diabetes mellitus: does gestational diabetes program Type 2 diabetes in the next generation? Diabet Med 23:1213-1215
44. De MM, Panico S, Iannuzzi A et al (2002) Association of obesity and central fat distribution with carotid artery wall thickening in middle-aged women. Stroke 33:2923-2928

45. Kanjilal S, Gregg EW, Cheng YJ et al (2006) Socioeconomic status and trends in disparities in 4 major risk factors for cardiovascular disease among US adults, 1971-2002. Arch Intern Med 166:2348-2355 